\title{
Research on the Relationship between Resilience and Mental Health of Boarders in Aba Tibetan and Qiang Autonomous Prefecture
}

\author{
Yining Wang \\ School of Computer Science and Technology \\ Southwest University of Science and Technology \\ Mianyang, China
}

\author{
Qian Xie \\ Department of Psychology \\ Southwest University of Science and Technology \\ Mianyang, China
}

\author{
Xieping Chen \\ Department of Psychology \\ Southwest University of Science and Technology \\ Mianyang, China
}

\begin{abstract}
Objective: examine the general situations of boarder's resilience and mental health, and the relevance between resilience and mental health to provide some theoretical suggestions for the psychological education in Aba prefecture. Methods: A total of six hundred and twenty-seven primary school students and junior school students in Song pan country of Aba Tibetan and Qiang Autonomous Prefecture were selected and assessed with the MMHI-Sixty for mental health, and the CD-RISC (ConnorDavidson Resilience Scale, (Connor-Davidson) for resilience. Results: the CD-RISC mean score was (three point two five plus or minus zero point five six), and each factor's mean scores were higher than three. It indicates that the level of resilience is optimistic. The mean score of MMHI-Sixty was two point six five plus or minus zero point six three), and the mild problem incidence rate was sixty five point one six percentage; the moderate problem incidence rate was nine point seven nine percentages. The problem incidence rate of factor is higher relatively, among those factors the obsession was eighty-eight point eight percent. The anxiety was seventynine point six four percent; the study pressure was seventyseven point eight percent. The resilience is remarkably negatively correlated with MMHI ( $p$ value are less than zero point zero one). Hierarchy regression analysis shows that resilience was significantly predictors of mental health, uniquely explaining eight point three percent. Discussion: the resilience was significantly negatively related with mental health of boarders in Aba prefecture, we could improve the level of resilience to make a difference on the degree of mental health on the psychological education in Aba prefecture.
\end{abstract}

Keywords-Aba prefecture; boarders; resilience; mental health

\section{INTRODUCTION}

Aba prefecture is located in the northwest of Sichuan province, which is the second largest Tibetan area in Sichuan province and the main area of Qiang ethnic in China, which owns the same natural characteristics as other minorities that harsh climate, scarce resource and their residential quarters are scattered and etc. The school is far away from home, and after undergoing a series of policies implementation on dismantling teaching points and combining schools, it has built a large scale of boarding schools, the boarders are far away from parental communication so that it is inevitable to reproduce some psychological problems for them. The relative research indicates that compared with the nonboarders, the level of mental health of boarders is lower than that of boarders, they could experience more negative emotion, such as loneliness, anxiety, depression and etc.

With the rising of positive psychology, positive traits are focused more and more by researchers. The positive psychology maintains that the measurable positive quantity could play a moderating and protective role when the individual are facing with the dangerous factors and environment. While the resilience belongs to the psychological traits, it means the mental development phenomenon when the individual are faced with the serious pressure adversity, their psychological function and development could far away from the damaging influence. Many scholars at home and abroad made beneficial explorations about resilience, it shows that the resilience were significantly positive related with mental health, the meaning are embodied in the protective effect on mental health. The higher level of resilience is the higher degree of mental health.

So far, there are many surveys about non-multinationality boarders and other ethnic areas boarders, and there are lack of investigations relatively on relationship between resilience and mental health of boarders in Aba prefecture. Therefore, our research selected the boarders in Songpan country Aba prefecture as the objective to explore the association between resilience and mental health, so that we can draw some scientific and valid conclusions on the 
psychological education. We hope it would provide beneficial advice.

\section{METHODS}

\section{A. Participants and Sampling}

Firstly, methods with stratified cluster randomly sampling methods, Song pan country was chosen from the Aba Prefecture, secondly, four townships of Song pan country and one school from each selected township (Songpan middle school, Hongtu villgage school, Xiao xing villgage school and Zhen Jiangguan middle school) were selected as sampling areas, then four to nine grades were randomly from each school, a total of 13 classes with 627 students were selected to do the questionnaires. It have been got 511 valid questionnaires (valid return rate is $81.5 \%$ ), and made up of boys 249 (the proportion is $48.7 \%$ ) and girls 262 (the proportion is $51.3 \%$ ), their average age is $(13.18 \pm 1.36)$ years.

\section{B. Measurement}

The CD-RISC scale was designed by psychologists Connor and Davidson in 2003, which contains 25 items including three dimensions: tenacity, strength and optimism that followed by domestic scholars who are Zhang Jianxin and Yu Xiaonan Each item including five answers choices ranging from "Not at All True" to "Very Much True", scoring1, 2, 3, 4, or 5, respectively. The higher scores the higher degree of resilience, the scale internal consistency reliability of our study is 0.89 .

The MMHI-60 scale was invented by domestic psychologist Wang Jishen, which was widely used to assess the mental health of middle school students in china, the scale consists of 60 items including ten content subscales, and each item was scored 1 for "never" and 5 for "yes", respectively. The higher score is, the higher degree of mental health problem is, and the worse the children's mental health status is. The scale was classified into mild levels (scoring below2), medium levels (between 2 and 3), heavier levels (between 3 and 4) and serious level (scoring is 5). The scale internal consistency reliability of our study is 0.94 .

Above all scales were revised properly under the condition of keeping the scale's original intention according to some feedback of pre-survey, so that it accord with the cognition of subjects.

\section{Procedure and Data Analysis}

The survey was conducted by 2 post-graduates who major in psychology and many teachers in November, 2016, all of who were trained in interviewing and administrating prior to investigation. All the participants were asked to complete the questionnaire independently and factually, if the children can't understand the meaning, they could hands up for help. After finishing the work above, the interviewers collected and checked all the questionnaires to make sure that there were no missing data or logical errors. And then the data were entered using Excel version2007, finally importing all the statistics to SPSS version 17, calculating the general information, the relevance about variables, and regression coefficients, The two-tailed $\mathrm{P}$ value of $<0.05$ were considered to be significant.

\section{RESULTS}

\section{A. The General Status of Boarders 'Resilience in Aba Prefecture}

In order grasp the level of resilience of boarders we calculate the mean scores (including three factors of resilience, and primary school boarders and middle school boarders). And then we conducted the independent sample T test to examine the difference in level of resilience between primary school boarders and middle school boarders in "Table I" as followed.

TABLE I. THE GENERAL SITUATION OF RESILIENCE AND THE INDEPENDENT SAMPLE T TEST FOR GRADES

\begin{tabular}{cllll}
\hline & Tenacity & Strength & Optimism & $\begin{array}{c}\text { Resilience } \\
\text { average }\end{array}$ \\
\hline $\begin{array}{c}\text { Primary } \\
(\boldsymbol{M} \pm \text { SD })\end{array}$ & $3.17 \pm 0.61$ & $3.25 \pm 0.66$ & $2.99 \pm 0.65$ & $3.13 \pm 0.53$ \\
$\begin{array}{c}\text { Middle } \\
(\boldsymbol{M} \pm \boldsymbol{S D})\end{array}$ & $3.30 \pm 0.66$ & $3.43 \pm 0.66$ & $3.17 \pm 0.95$ & $3.29 \pm 0.56$ \\
$\boldsymbol{T}$ value & -1.96 & -2.52 & -2.28 & -2.69 \\
$\boldsymbol{P}$ value & $>0.05$ & $<0.05$ & $<0.05$ & $<0.01$ \\
\hline
\end{tabular}

We can see from the above table (mean scores) that the boarders' level of resilience are in the higher medium level (the medium value equals three). Meanwhile, the independent sample $\mathrm{T}$ test shows that the general level of resilience of primary school boarders is lower than that of middle school boarders. ( $\mathrm{P}$ value is lower than zero point zero one), but there was no significant difference in the factor of tenacity between the primary school boarders and middle school boarders.

\section{B. The General Situation of Boarders' Mental Health in Aba Prefecture}

We calculate the total average and ten factors' mean score of MMHI, and then we perform the single-test T test with norm of the Chinese middle student in MMHI. The result shows that there are remarkable difference between boarders' level of mental health and that of Chinese middle school students ( $p$ values are all lower than zero point zero one), the further displayed that the level of mental health of boarders in Aba prefecture is lower significantly than that of Chinese middle school students as followed by "Table II". 
TABLE II. The SingLE-SAMPLE T TEST BETWEEN THE BoARDERS AND CHINESE MidDLE SCHOOL STUDENTS NORM IN MMHI $(\mathrm{M} \pm \mathrm{SD})$ $(\mathrm{N}=511)$

\begin{tabular}{|c|l|l|l|}
\hline & $\begin{array}{c}\text { Chinese } \\
\text { student's norm }\end{array}$ & $\begin{array}{c}\text { The boarders in } \\
\text { Aba prefecture }\end{array}$ & \multicolumn{1}{|c|}{ T value } \\
\hline Obsession & $1.89 \pm 0.61$ & $2.63 \pm 0.59$ & $28.72^{* * *}$ \\
\hline Stubborn & $1.76 \pm 0.86$ & $2.40 \pm 0.66$ & $21.84^{* *}$ \\
\hline Hostility & $1.72 \pm 0.75$ & $2.27 \pm 0.76$ & $16.38^{* * *}$ \\
\hline $\begin{array}{c}\text { Interpersonal- } \\
\text { relationship }\end{array}$ & $1.85 \pm 0.73$ & $2.29 \pm 0.66$ & $15.00^{* * *}$ \\
\hline Depression & $1.92 \pm 0.67$ & $2.22 \pm 0.68$ & $9.59^{* * * *}$ \\
\hline Anxiety & $1.81 \pm 0.75$ & $2.48 \pm 0.74$ & $20.46^{* * *}$ \\
\hline Study pressure & $1.92 \pm 0.67$ & $2.56 \pm 0.79$ & $18.30^{* * *}$ \\
\hline Maladjustment & $1.92 \pm 0.62$ & $2.15 \pm 0.69$ & $7.50^{* * *}$ \\
\hline $\begin{array}{c}\text { Emotional- } \\
\text { imbalance }\end{array}$ & $1.98 \pm 0.70$ & $2.39 \pm 0.64$ & $14.53^{* * *}$ \\
\hline $\begin{array}{c}\text { Mental- } \\
\text { imbalance }\end{array}$ & $2.02 \pm 0.62$ & $2.35 \pm 0.65$ & $2.60^{* * *}$ \\
\hline Total & $1.88 \pm 0.57$ & $2.34 \pm 0.47$ & $22.11^{* * *}$ \\
\hline
\end{tabular}

a. Note:* means $p<0.05, * *$ means $p<0.01, * * *$ means $p<0.001$, the same below
The testing results shows that the mean score of MMHISixty was (two point six five plus or minus zero point six three), and the mild problem incidence rate was sixty five point one six percentage; the moderate problem incidence rate was nine point seven nine percentage. The problem incidence rate of factor is higher relatively, among those factors the obsession was eighty-eight point eight percent. The anxiety was seventy-nine point six four percent; the study pressure was seventy-seven point eight percent.

\section{The Relevance Testing between Resilience and Mental Health of Boarders in Aba Prefecture}

The analysis of Pearson's correlation coefficient between mental health and resilience shows that all the factors in MMHI were significantly negatively correlated with resilience except for the factors (obsession and stubborn) as followed by "Table III".

TABLE III. The ReleVAnCE BetweEn Resilience And Mental Health (R VALUE, N=511)

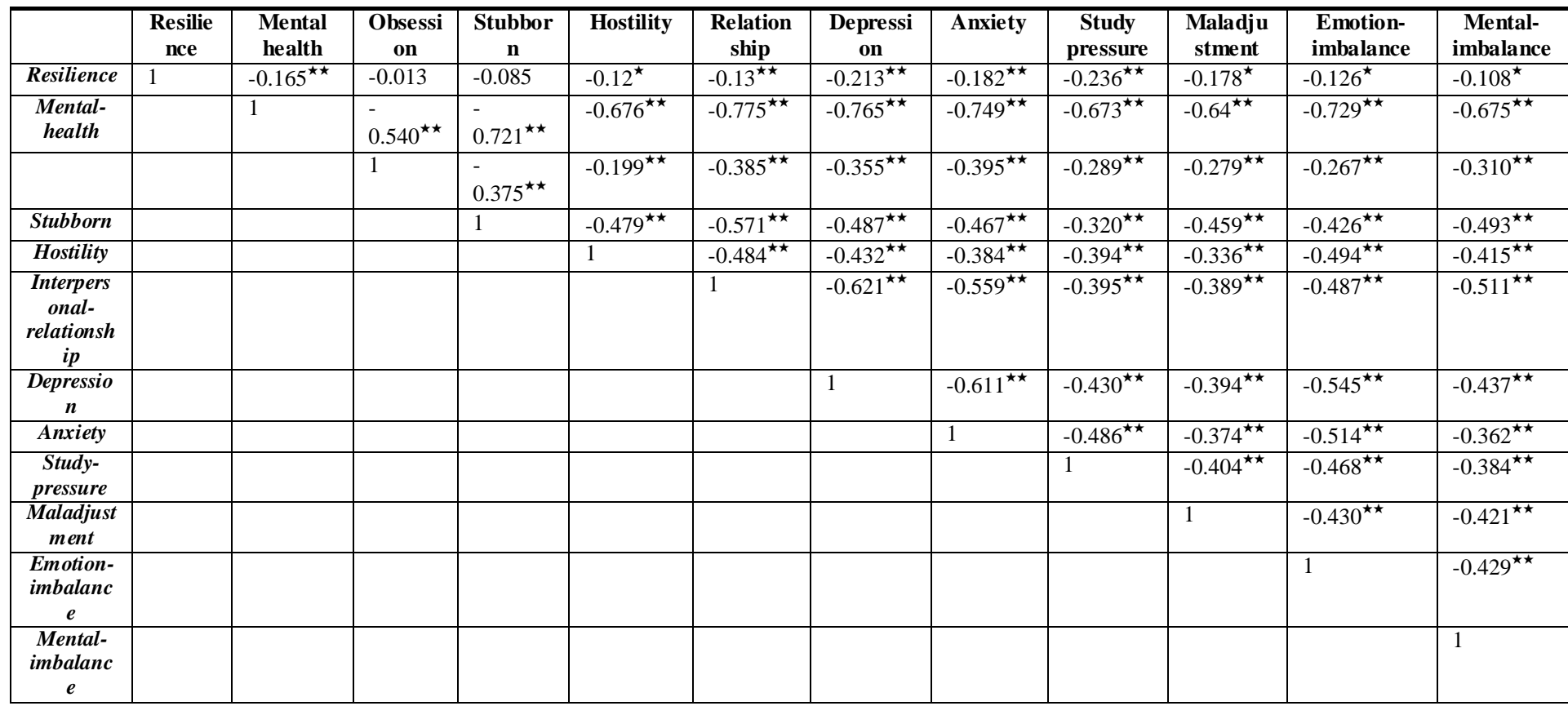

\section{Hierarchy Regression Analysis of Mental Health and Resilience}

Our study used the hierarchy regression analysis to examine whether the resilience could exert a predicting effect on mental health under the control of grade and gender.

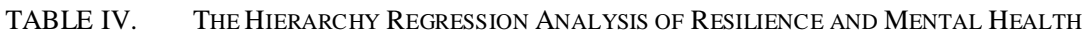

\begin{tabular}{|c|c|c|c|c|c|c|c|}
\hline & & $\begin{array}{c}\text { Anxiety } \\
\text { First step }\end{array}$ & $\begin{array}{c}\text { Second } \\
\text { step }\end{array}$ & $\begin{array}{l}\text { Depression } \\
\text { First step }\end{array}$ & Second step & $\begin{array}{c}\text { Study-pressure } \\
\text { First step }\end{array}$ & Second step \\
\hline \multirow[t]{2}{*}{ First step } & gender & 0.088 & 0.109 & 0.055 & 0.074 & 0.101 & 0.111 \\
\hline & grade & 0.024 & 0.031 & 0.018 & 0.024 & 0.034 & 0.037 \\
\hline \multirow[t]{6}{*}{ Second step } & tenacity & - & $-0.091^{* *}$ & & $-0.026^{* *}$ & & -0.014 \\
\hline & strength & & $-0.183^{* *}$ & & $-0.094^{* *}$ & & $-0.032^{*}$ \\
\hline & optimism & & $-0.046^{* *}$ & & -0.016 & & $-0.053^{* *}$ \\
\hline & $\Delta F$ & 1. 658 & 3. $557^{* *}$ & 0.905 & 4. $753^{* *}$ & 3. 357 & $1.960^{*}$ \\
\hline & $R 2$ & 0.007 & 0.027 & 0.004 & 0.031 & 0.013 & 0.025 \\
\hline & $\Delta R 2$ & 0.007 & 0.021 & 0.004 & 0.028 & 0.013 & 0.011 \\
\hline
\end{tabular}


Resilience is considered to be the independent variable and mental health is considered to be the dependent variable. Because the factors of anxiety, depression and study-pressure are in most remarkable negative correlated with the resilience, we selected the three major factors to enter into regression formula. Firstly, the demographic variable (grades and gender) are included in the first layer regression equation, and then make the resilience as the second one enter into the regression equation to calculate the variation of $\mathrm{R} 2$ between the two layers and the last results as followed by "Table IV".

From the above table, we know that the resilience exert a remarkable negative predicting influence on the factors of anxiety, depression and study pressure. It means the amount of added explanatory variable are $2.1 \%, 2.8 \%$ and $1.1 \%$ respectively.

\section{CONCLUSION}

Our study shows that the average score of resilience was (3.25 \pm 0 . 56), and the single-sample $\mathrm{T}$ test indicates the average scores have a significant difference with the medium value 3 , which indicates the situation of resilience is optimistic. The probable reason are that the specific geographic conditions, ethnic culture and religious faith make the local people stronger inner quality when encounter the adversity. On the other hand, natural disasters frequently occur in Aba prefecture such as earthquake, mud-rock flow and landslide etc., which make the local people experienced more disaster events than others, so they develop stronger and tenacious personalities, those characters are the foundation of high level of resilience. Meanwhile, we found that the level of resilience of middle school boarders is higher than that of primary school boarders, we believe that as the grow of age the development of physical and mental is improving, what's more, the middle school boarders suffering more life events so they own the abilities to deal with the difficulties to conquer the fragility and sensitivity. Those results are corresponding with the previous research.

Our findings indicates that level of the mental health of primary boarders is significantly lower than that of middle school boarders, it means that as the age increased, the average MMHI-60 scores of the boarders were remarkably declined, which was accordance with the other studies focus on boarders and left-behind children. on the one hand, the primary boarders' scores of maladjustment is higher than that of middle school boarders, The longer time of boarding life and physical and mental development is growing, they are in social development stage to formulate the quality of cooperation, self-discipline and freedom, so they own a relatively psychological environment to accommodation in school. However, the study pressure scores of middle boarders' are higher than primary school boarders. Analysis the reasons for this findings, it probably due to under the traditional nation's oriented system, the middle school student have to be faced with more powerful enrollment pressure and fierce competition. Besides, the boarders in Aba prefecture have a long for walking out mountain to look for outside world and broaden their horizons, perhaps, this is the reliable way to help them realize their dream, studying hard is an invisible pressure for them. But for primary boarders, they still have not built this cognition and awareness, so their study pressure status is better. What else, because the high altitude and climate in Aba prefecture and the sports facilities isn't enough in schools, directly related to the effect of the physical and mental psychological training of boarders who have high study pressure and other mental health problems. What's worse, the school have not been the relatively improving psychological education system makes the boarders whose mental problems being the free and ignored status, as time goes by, their psychological problems . Hierarchy regression and relevance analysis of mental health and resilience shows that the resilience have a significantly negative predicting effect on individual mental health, it means the higher level of resilience the higher degree of mental health . It suggests that we could improve the level of resilience from the positive psychology perspective.

\section{REFERENCES}

[1] Zhang H. (2010). The research on mental health of primary and middle school boarders. (Doctoral dissertation, china medical university).

[2] Wang DT, Zhang XZ, Hen WJ. (2014). The investigation on mental health of middle school boarders in rural areas. Academic magazine of He xi college, 126-128.

[3] Zhi YL, (2013). The investigation on mental health of primary school middle school boarders in rural areas. Academic magazine of Jin zhong college, 83-86.

[4] Rutter, M. (1993). Resilience: some conceptual considerations Journal of Adolescent Health Official Publication of the Society for Adolescent Medicine, 14 (8), 690-6.

[5] Wang YH. (2010). Research on social support net of left-behind children from the perspective of resilience. Education academic monthly. 10 (6-7)

[6] Betancourt, T. S., \& Khan, K. T. (2008). The mental health of children affected by armed conflict: protective processes and pathways to resilience. International Review of Psychiatry, 20 (3), 317-328.

[7] Davydov, D. M. , Stewart, R. , Ritchie, K. , \& Chaudieu, I. (2010). Resilience and mental health. Clinical Psychology Review, 30 (5), 479.

[8] Connor, K. M. , \& Jonathan, R. T. D. M. D. (2003). Development of a new resilience scale: the connor-davidson resilience scale (cd-risc). Depression and Anxiety, 18 (2), 76.

[9] Wang JS, Li Y, (1997). the compontent and standardization of mental health scale of chinese middle school students. Science of Social Psychology (4), 21-25

[10] Wang YH. (2010). Studying the left -behind children's social support net from the perspective of resilience. Educational Research Monthly (10), 6-7.

[11] Zhou YH, Lv CF, \& Xu FH. (2013). The study between resilience and mental health in left-behind children. Chinese Journal of Special Education (10)

[12] Jiang SK, \&Liang JL. (2015). The investigation study of mental health status between boarders and non-boarders. The Academic Journal of Xiang Y yang Professional Technology College (2), 121125

[13] Li KY. (2014). The relationship study about loneliness mental health and social support of middle boarders. (Doctoral dissertation, Chong Qing Normal University).

[14] Yang XJ. (2010). The relationship study between school adjustment and mental health of middle boarders in Yan bian areas. (Doctoral dissertation, Yan bian university) 